\title{
SMALL BOWEL IMAGING - STILL A RADIOLOGIC APPROACH?
}

\author{
Ingrid Markova, ${ }^{\mathrm{a}, \mathrm{*}}$, Katerina Kluchova ${ }^{\mathrm{b}, \mathrm{d}}$, Radek Zboril ${ }^{\mathrm{b}, \mathrm{d}}$, Miroslav Mashlan ${ }^{\mathrm{b}}$, \\ Miroslav Herman ${ }^{c}$
}

\author{
a Department of Radiology, Faculty Hospital F. D. Roosevelt Banska Bystrica, Slovak Republic \\ b Centre for Nanomaterial Research, Palacky University Olomouc, Czech Republic \\ Department of Radiology, Faculty of Medicine and Dentistry, Palacky University \\ d Department of Physical Chemistry, Palacky University \\ E-mail:ingrid.markova@gmail.com
}

Received: September 3, 2009; Accepted: May 28, 2010

Key words: CT enteroclysis/MR enteroclysis/Crohn's disease/Small bowel neoplasm/Contrast- enhanced ultrasound/Super Paramagnetic of Iron Oxides (SPIO)

Background. In recent years, there has been renewed interest in small bowel imaging using a variety of radiologic or endoscopic techniques. This article gives an overview and comparison of old and new techniques used in small bowel imaging. New imaging methods as computed tomography (CT), CT enteroclysis (CTEc), CT enterography (CTEg), ultrasound (US), contrast-enhanced ultrasound (CEUS), US enteroclysis, US enterography, magnetic resonance imaging (MRI), MR enteroclysis (MREc) and MR enterography (MREg) are compared with the older techniques such as small- bowel follow- through (SBFT), conventional enteroclysis (CE) and endoscopic techniques including push enteroscopy, ezofagogastroduodenoscopy (EGD), sonde enteroscopy, ileocolonoscopy, double-balloon enteroscopy, intraoperative enteroscopy and wireless capsule enteroscopy (WCE).

Methods. Systematic scan of Pubmed,Medline, Ovid, Elsevier search engines was used.. Additional information was found through the bibliographical review of relevant articles.

Results. SBFT has only secondary role in small bowel imaging. US is still the method of choice in imaging for pediatric populations. US and CEUS are also accepted as a method of choice especially in inflammatory cases. CE has been replaced by new cross - sectional imaging techniques (CTEc/CTEg or MREc/MREg). CTEc combines the advantages of CT and CE. MREc combines the advantages of MRI and CE. Some authors prefer CTEg or MREg with peroral bowel preparation and they strictly avoid nasojejunal intubation under fluoroscopic control. MREc has better soft tissue contrast, showing it to be more sensitive in detecting mucosal lesions than CTEc in inflammatory diseases. CTEg/MREg are techniques preferred for patients in follow-up of the inflammatory diseases. The radiologic community is not unanimous however about their role in the imaging process. CTEc/MREc as well as CTEg/MREg are superior to endoscopic methods in the investigation of small-bowel tumors. WCE gives unparalleled imaging of the mucosal surface of the small bowel especially in the event of obscure gastrointestinal bleeding and inflammatory diseases.

Conclusions. In a comparison of endoscopic and radiologic approaches, radiologic techniques are less invasive for patients, they take less time to investigate and allow imaging the entire small bowel. Some do not involve radiation exposure (US, MR). Endoscopic methods are more expensive, more invasive, need longer examination time and technical special skills but without radiation exposure. The greatest advantage of some endoscopic methods is the possibility of mucosal biopsy in one step with diagnostic examination (EGD, push enteroscopy, intraoperative enteroscopy, ileocolonoscopy).

\section{INTRODUCTION}

There was a time when small-bowel follow-through (SBFT) was the primary method of diagnosing diseases of the small bowel. In recent years, there has been renewed interest in small bowel imaging using a variety of technique such as ultrasound(US) contrast enhanced ultrasound (CEUS), computed tomography (CT), magnetic resonance imaging (MRI), computed tomography enteroclysis/enterography (CTEc/CTEg) and magnetic resonance enteroclysis/enterography ( $\mathrm{MREc} / \mathrm{MREg}$ ) and the small bowel endoscopic methods. The introduc- tion of helical CT technology in 1989 and subsequently multidetector CT has further changed small bowel imaging. CT has faster acquisition of a large volume of data with thinner collimation and possibility of high-quality multiplanar reformatting. The introduction of MRI has created an important tool for small bowel imaging too. MRI has the ability of increased soft tissue contrast without ionizing radiation and using ultrafast sequences. The limitations of push enteroscopy and ileocolonoscopy allowed the dominance of radiologic methods in the workup of patients with small bowel diseases. Wireless capsule enteroscopy (WCE) is utilized in the elective investiga- 
tion of small bowel diseases. Current clinical guidelines recommend the use of radiological studies prior to use of WCE especially in high risk patients ${ }^{1}$. Digital subtraction angiography (DSA) for diagnostic purposes has in the majority of indications been replaced by CT angiography (CTA) while DSA is reserved for guidance of interventional procedures. New ultrafast techniques in MRI and magnetic resonance angiography (MRA) using i.v. contrast medium are able to compete with CTA. The latter method is ultrasound assessment of the small bowel without radiation exposure. Always challenging because of the gas content, this method is favoured in pediatric gastroenterology. Ultrasound needs experienced sonographers and good cooperation with clinicians.

\section{Radiologic imaging techniques}

Small-bowel follow-thsrough (SBFT). Historically SBFT has been the standard radiologic approach used to asses patients with gut diseases. The patient drank 500-600 ml of barium suspension after fasting for at least 8 hours. Serial overhead radiographs were obtained in prone position at 30 to 60 minute intervals until the barium reached the ileocaecal valve. SBFT provided information about intraluminal disease extension and small bowel motility disorders. Higher radiation dose and no informations on extramural disease extention caused its replacement by modern cross-sectional imaging methods.

Conventional enteroclysis (CE). The principle of this method is the administration of nasojejunal tube under fluoroscopic guidance, getting the tip of tube right in the duodenal-jejunal flexure. The administration of

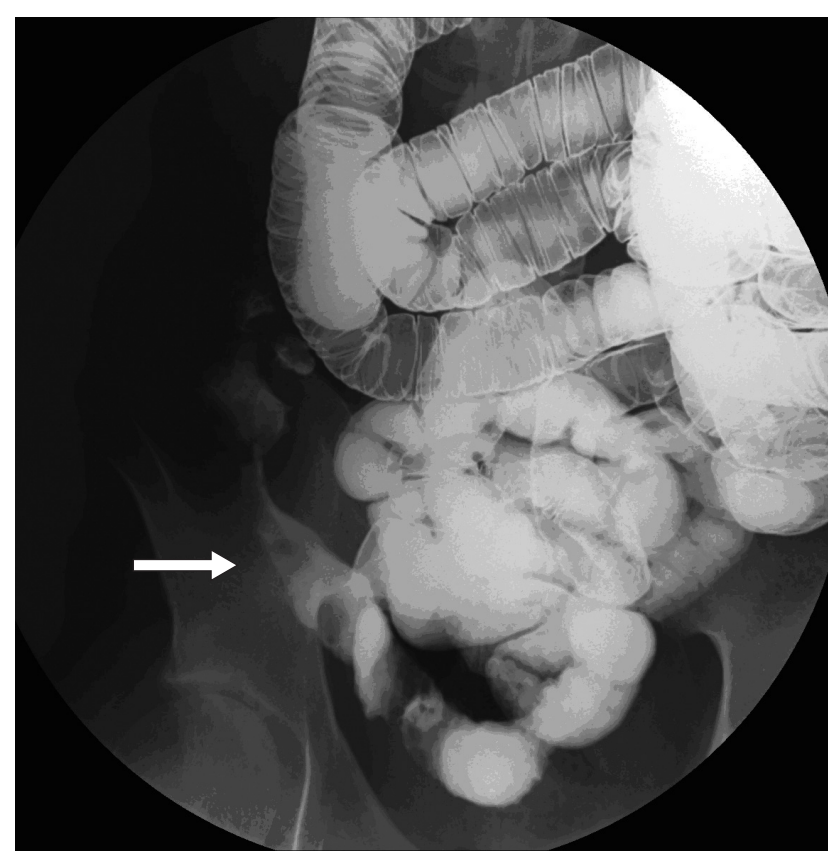

Fig. 1. Active ileal Crohn's disease in 21-year-old woman. Conventional enteroclysis with positive luminal filling (barium + Vidogum) reveals luminal narrowing (arrow) in terminal ileal loop, oral ileum and jejunum are without disease.
200-250 $\mathrm{ml}$ barium sulphate suspension followed by $0.5 \%$ metylcellulose or Vidogum (Vidogum GH 250, CO ORD-Med,CZ) through the nasojejunal tube is performed manually or by automatic pump in total volume of 1500-2000 ml. The balloon can be administered to the duodenum to prevent of reflux into the stomach. The aim of the procedure is to reach a complete distension of the entire small bowel loops. After adequate demonstration of ileocaecal transition, posterio-anterior and oblique film radiographs are obtained and at the end the compression spot views of distal ileum are done (Fig. 1). Duration of the examination, discomfort due to intubation and infusion of large volumes of contrast and distension materials lead to the low acceptance of this method ${ }^{1}$. CE has been shown to be highly accurate, with a sensitivity of $93.1 \%$ and a specificity of $96.9 \%$ (ref.$^{2}$ ) in diagnosing small bowel diseases, and permits detection of partly obstructive or nonobstructive lesions that may not be demonstrated with cross-sectional imaging techniques. The principal disadvantage is the limited indirect information on the state of the bowel wall and extramural extension, and its effectiveness may be hindered owing to overlapping bowel loops. The radiation dose administered to young patients should also be considered ${ }^{3}$. On the other hand, competing cross-sectional methods are not able to assess superficial mucosal lesions in inflammatory diseases in such details ${ }^{4,5}$ . CE is the most efficient diagnostic modality in evaluating cases of postoperative obstruction - etiology, location and determination the degree of obstruction ${ }^{6}$.

Ultrasound, contrast-enhanced ultrasound (CEUS), new ultrasound imaging techniques. Imaging of small bowel diseases with ultrasound is always challenging. Routine sonograms are performed with real time $3.5-5 \mathrm{MHz}$ transducers. Areas of interest are analysed with compression sonography. The sonographer must look for "target pattern" and "pseudokidney pattern", both of which correspond to thickened gut wall. Gut wall masses may be intraluminal, mural or exophytic, all with or without ulceration. Intraluminal gut masses are frequently hidden by gas or luminal content. More readily visualised are exophytic masses, the problem is to assign them a gastrointestinal tract origin, if typical gut patterns (target or pseudokidney) are not seen on ultrasound. Intraperitoneal masses of varying morphology, which do not clearly arise from the solid abdominal viscera or the lymph nodes, should be considered to have a potential gut origin.

Classic sonographic features in Crohn's disease are wall thickening, strictures, mesenteric fat creeping, hyperemia, mesenteric lymphadenopaty. Mucosal abnormalities and complications like inflammatory masses, fistula, obstruction, perforation and appendicitis are also seen. Luminal dilatation, intensive peristaltic waves, wall thining, above the stricture are seen in moderate and severe stages of Crohn's disease. Mesenteric fat creeping is the most common cause found to explain bowel loop separation in Crohn's disease as well as other forms of inflammatory bowel diseases (IBD). Doppler evaluation with or without intravenous (i.v.) contrast medium can differentiate inflammatory from ischemic bowel wall thickening?. 
Ultrasound (US) examination seems to be the best method for screening and is preferred method in pediatric population. Mesenteric lymphadenitis and acute ileitis is the most frequent GI cause of misdiagnosis of acute appendicitis especially in pediatric population, conventional US can help in differentiation between them. Recently by using of the so-called US angiography it has become possible to evaluate vascularization of the reinforced wall of the affected portion of the small intestine as well and thus also the activity of the inflammation ${ }^{8}$. Contrast - enhanced ultrasound (CEUS) is a special part of ultrasound chapter. Untargeted CEUS $^{9}$ uses untargeted microbubbles. Microbubbles are injected intravenously into the systemic circulation in a small bolus. The microbubbles will remain in the systemic circulation for a certain period of time. During that time, ultrasound waves are directed to the area of interest. When microbubbles in the blood flow past the imaging window, the microbubble's compressible gas cores oscillate in response to the high frequency sonic energy field. The microbubbles reflect a unique echo that stands in stark contrast to the surrounding tissue due to the orders of magnitude mismatch between microbubble and tissue echogenicity. The ultrasound system converts the strong echogenicity into a contrast-enhanced image of the area of interest ${ }^{9}$. Targeted CEUS works in a similar fashion, with a few alterations. Microbubbles linked with ligands that bind certain molecular markers, are expressed by the area of imaging interest.Targeted microbubbles are injected intravenously in a small bolus. Microbubbles theoretically travel through the circulatory system, eventually finding their respective targets and are bound specifically9.

US enteroclysis using tap water and filling small bowel through nasojejunal tube, seems to have become the standard examination of patients with Crohn's disease mainly in those with unclear conventional ultrasound ${ }^{10}$. The most important fact is that this examination significantly decreases the radiation dose while maintaining high sensitivity. This is very important in patients with Crohn's disease who require repeated examinations lifelong examinations. This examination is much easier to standardize than the conventional US ${ }^{10}$.

US enterography with small bowel prepared by drinking of 500-1000 $\mathrm{ml}$ of the water has better patient acceptance in follow-up of inflammatory disease.

The role of sonography in the evaluation of GI tract neoplasms is similar to that of CT scan. Sonograms are frequently performed early in the diagnostic work- up of patients with GI tract tumors, often before their initial identification. Appreciation of the typical morphologies associated with tumors may lead to accurate recognition, localization and even staging of disease with the opportunity for directing appropriate further investigation, including sonography - guided aspiration biopsy?

CTenteroclysis ( CTEc), CTenterography (CTEg). CT has become a routine examination in the evaluation of gastrointestinal disorders because of rapid execution, its accuracy with axial images and multiplanar reconstruction. Multislice CT has improved image quality and has reduced scan times. Primary requirements of CT examination should be pursued: visualisation of the entire small bowel, adequate visceral distension, elimination of respiratory motion and peristalsis, intravenous administration of contrast agent to evaluate the extent and patterns of wall enhancement. 12 hours fasting before examinaton is needed. Abdominal CT without enteroclysis/enterography is performed after ingestion of positive (iodine solution or barium solution) or neutral (water) contrast agent within 1,5-2 hours before examination. Postprocessing uses multiplanar reconstruction (MPR), maximum intensity projection (MIP) and three-dimensional volume rendering (3D VR) techniques. CT enteroclysis (CTEc) combines the advantages of $\mathrm{CT}$ and $\mathrm{CE}$ into one technique ${ }^{11}$. CTEc can be performed by using either positive enteral contrast agent without intravenous contrast agent or neutral enteral contrast agent with intravenous contrast agent ${ }^{12}$. With either technique, bowel preparation includes low residue diet 24 hours before examination and 6-12 hours fasting before the examination. The placement of a 12-16 F nasojejunal tube under fluoroscopic control is needed with or without conscious sedation and analgesia. In case of positive enteral contrast agent the administration of contrast material (iodine or barium solution) (ref. ${ }^{12}$ ) is performed through the nasojejunal tube either manually or by automatic pump in total volume of 1500-2000 ml, with an infusion rate of $55-150 \mathrm{ml} /$ minute. For reducing abdominal discomfort and avoiding spasm, a spasmolytic agent (butylscopolamin or glucagon) is administered intravenously. In case of neutral contrast agent in CTEc, the administration of VoLumen (E-ZEM/Bracco-low concentration of barium solution containing sorbitol) (ref..$^{13}$ ) or polyethylenglycol (PEG) or Vidogum (Vidogum GH 250, CO ORD-Med,CZ) or water in a total volume 1500$2000 \mathrm{ml}$ ( ref. $^{12}$ ). Rate infusion the same as in positive enteral contrast agent is performed. Regardless of the kind of enteric contrast agent, the CT scan is done 50 seconds after intravenous administration of $150 \mathrm{ml}$ of contrast agent, at a rate $4 \mathrm{ml} / \mathrm{s}$, total $150 \mathrm{ml}\left(\right.$ ref. $^{12}$ ). Hypodense contrast medium ensures better definition of the internal aspect of the small bowel, especially to evaluate the degree of parietal contrast enhancement after intravenous infusion contrast bolus ${ }^{11}$. The advantages of water include lower cost and reduced viscosity, which allows a smaller nasoenteral tube or faster infusion rates in $\mathrm{CTEc}^{11,13}$. In CT the normal thickness of the intestinal wall is barely perceptible in a wall-distended segment and should be no greater than 2-3 mm thick: during i.v. administration of the contrast agent bolus, there is a normal enhancement of the bowel wall. Inadequate contrast opacification of the intestinal lumen and incomplete luminal distention accounts for the majority of errors.

CT enterography (CTEg) is an alternative technique to CTEc, where small-bowel filling is achieved with oral hyperhydration with at least $1500-2000 \mathrm{ml}$ of oral contrast agent. The choice of optimal enteric contrast agent is important. Contrast agent must not only allow detection of enhancing bowel wall abnormalities, it must also provide good luminal distension and be well tolerated by patients $^{14}$. In a prospective, randomized comparison of 
high-vs low- attenuation enteric contrast media in 90 consecutive patients without small-bowel disease, Erturk et al. concluded that low-attenuation (neutral) oral contrast media provided equal or superior distension and bowel wall visualization compared to the high-attenuation (positive) contrast medium. For these reasons, neutral enteric contrast agents are preferred when performing routine $\mathrm{CTEg}^{14}$. Neutral enteral contrast agents as water, PEG, VoLumen, 2.5\% solution of mannitol or sorbitol are well tolerated by patients ${ }^{14}$. Wold et al. ${ }^{15}$ found no significant difference in the adequacy of luminal distension between peroral water CTEg and CTEc. However, distention of the small bowel with CTEg may not be as well-guaranteed as that with CTEc. Patient discomfort associated with nasoenteric intubation, time-consuming examination, additional radiation exposure and requirement of technical skills, makes CTEg preferred by patients to $\mathrm{CTEc}^{14}$. In a European series ${ }^{16}$ on CTEg, 20 (19\%) of 106 study patients were removed from the analysis because of poor or absent small-bowel distention. In a prospective comparison between MR enteroclysis (MREc) and CTEc, the latter showed higher sensitivity and interobserver agreement for imaging signs of small-bowel disease ${ }^{15}$. The sensitivity of CTEc for bowel wall thickening, abnormal bowel wall enhancement, and adenopathy was $89 \%, 79 \%$, and $64 \%$, respectively. For the same signs, the sensitivity of MREc was $60 \%, 56 \%$, and $14 \%$, respectively. The interobserver agreement for these signs varied between 0.52 and 0.65 for CTEc and between 0.15 and 0.48 for $\mathrm{MREc}^{15}$. CTEc is the only study that has been found to reliably help exclude small-bowel strictures prior to capsule study. In recent study of 56 patients with known Crohn's disease, CTEc and wireless capsule enteroscopy (WCE) both depicted a substantial number of terminal ileal Crohn's lesions ( 24 with WCEvs 21 with CTEc), unlike those for jejunal lesions (25 with WCE vs 12 with CTEc) ${ }^{17}$. CTEc has been of value in resolving the false-positive and falsenegative interpretations from nonenteral volume-challenged small-bowel studies that arise from the difficulties associated with poor distention or peristalsis, simulating wall thickening ${ }^{18}$. There are some disadvantages of CTEc - the discomfort associated with nasojejunal tube placement may be improved with the use of local sedation and smaller tubes. The logistics of performing CT when the fluoroscopic suite is far from the CT area is inconvenient. The use of CT units with fluoroscopic capabilities may be more helpful when neutral enteral contrast material is used for enteroclysis. Although CTEc requires additional time for tube placement, the time for interpretation of this examination is less than that required for other complex CT studies. The addition of CT to enteroclysis increases the cost of the procedure, and exposure of patients and staff to radiation remains an important issue even if newer multidetector CT technology has improved dose efficien$\mathrm{cy}^{19}$. The invasiveness of the procedure raises concern for complications such as bowel perforation, enteral contrast material aspiration, or respiratory depression from sedation. In some cases, the procedure cannot be performed because of the inability to place the enteroclysis tube in an adequate position or lack of patient cooperation (pediat- ric population). CT or CTEg is recommended for revealing congenital lesions. In patients with Crohn's disease, CTEg is increasingly used to detect enteric inflammation in addition to extra-enteric complications ${ }^{15}$. Voderholzer et al. ${ }^{20}$ recently evaluated 56 patients with Crohn's disease who underwent CTEc and WCE and found that, for terminal ileal inflammation due to Crohn's disease, CTEc had a sensitivity of $67 \%$ and WCE had a sensitivity of $80 \%$. In a recent study comparing portal phase CTEg, WCE, SBFT and ileocolonoscopy, researchers reported sensitivities of $82 \%, 83 \%, 65 \%$, and $74 \%$, respectively, for active Crohn's disease but found that CTEg was more specific than $\mathrm{WCE}^{21}$. Findings such as mural thickening, mural enhancement, increased attenuation of the perienteric fat, and the "comb sign" have been reported to indicate active inflammatory Crohn's disease ${ }^{22}$. Booya et al. ${ }^{23}$ found that jejunal attenuation is significantly higher than ileal attenuation ( $113 \mathrm{HU}$ vs $72 \mathrm{HU}, p=0.001$ ) when examining distended small-bowel loops at enteric phase CTEg. Furthermore, collapsed jejunal and ileal loops have greater attenuation than their distended counterparts ( $p$ $=0.001)$. These small-bowel attenuation patterns are important for radiologists to recognize for evaluating the patients with active Crohn's disease, because segmental mural hyperenhancement at $\mathrm{CT}$ and magnetic resonance (MR) imaging is indicative of active inflammation ${ }^{24,25}$. The most sensitive visual CT finding of Crohn's disease activity was mural hyperenhancement, which had a sensitivity of $73-80 \%$ according to radiologist assessment ${ }^{24}$. Increased mural thickness had slightly lower sensitivity for active Crohn's disease, but was a more specific finding. The comb sign (Fig. 2) and increased attenuation in the perienteric fat were the most specific findings of active

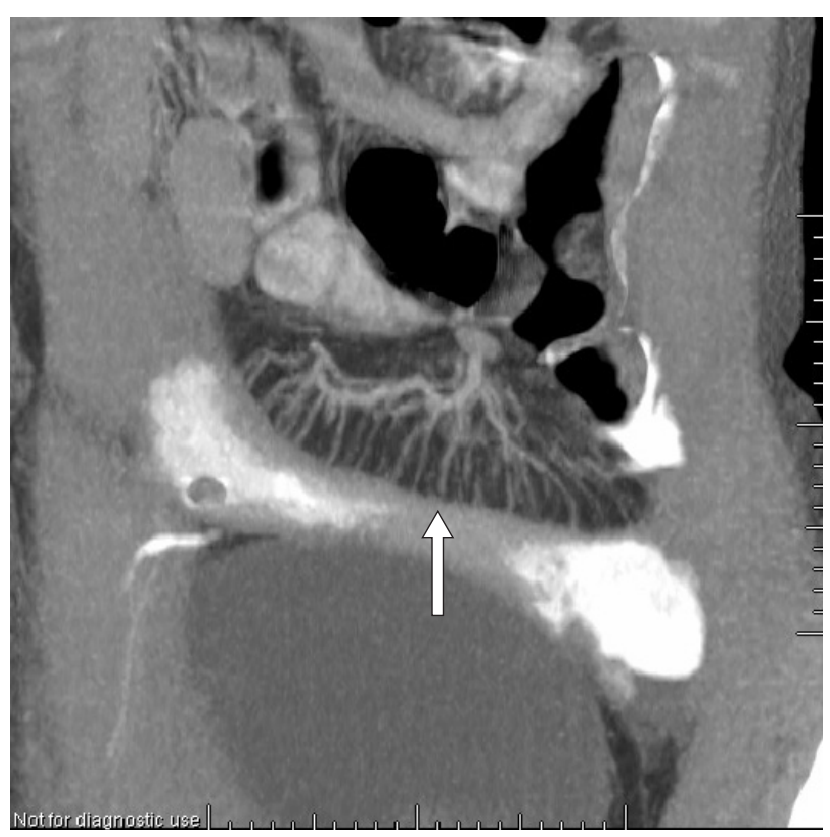

Fig. 2. Active ileal Crohn's disease in 33-year-old man. Coronal enteric phase CT enterography with positive luminal contrast agent, reveals luminal narrowing, mural thickening and the prominence of the mesenterial vessels "comb sign" (arrow). 
disease $^{24}$, while mucosal and submucosal abnormalities (eg, vascular malformations, nonsteroidal anti-inflammatory drug ulcerations, and neoplasms) may be optimally detected using invasive techniques such as WCE. Some studies found CTEg compared favorably with WCE in patients suspected of having Crohn's disease and finding that has also been suggested by other authors ${ }^{26-28}$. There is emerging evidence that CTEg has a large impact on clinical management decisions in patients with known and suspected Crohn's disease. Bruining and Fletcher ${ }^{14}$ found that CTEg findings correlated significantly better with disease presence than did clinical symptoms and serum markers of inflammation. In suspicion of Whipple's disease or celiac disease CTEc/ CTEg is also recommend$\mathrm{ed}^{29}$. A lot of reports have confirmed the effectiveness of CTEc in the diagnosis of small-bowel neoplasmsref. ${ }^{13,30}$ because of its ability to characterize the small-bowel wall, its mesentery and the liver. More accurate determination of primary or metastatic lesions involving the small bowel is possible with CTEc compared with conventional (nonenteroclysis) CT (Fig. 3). However, if the indication is pain or small-bowel obstruction, CT will usually be the first-line examination $^{28,31-33}$. Although the accuracy of multidetector CT with neutral oral and intravenous contrast material has improved, the detection of small nonobstructive primary tumors requires optimal bowel distention that is achievable with CTEc. CTEc has a complementary role to WCE and push enteroscopy in screening for small polyps in familiar polyposis and Peutz-Jeghers syndromes.

$M R$ enteroclysis ( $M R E c), M R$ enterography (MREg). Magnetic resonance imaging (MRI) is the preferred diagnostic procedure, due to the absence of ionizing radiation,

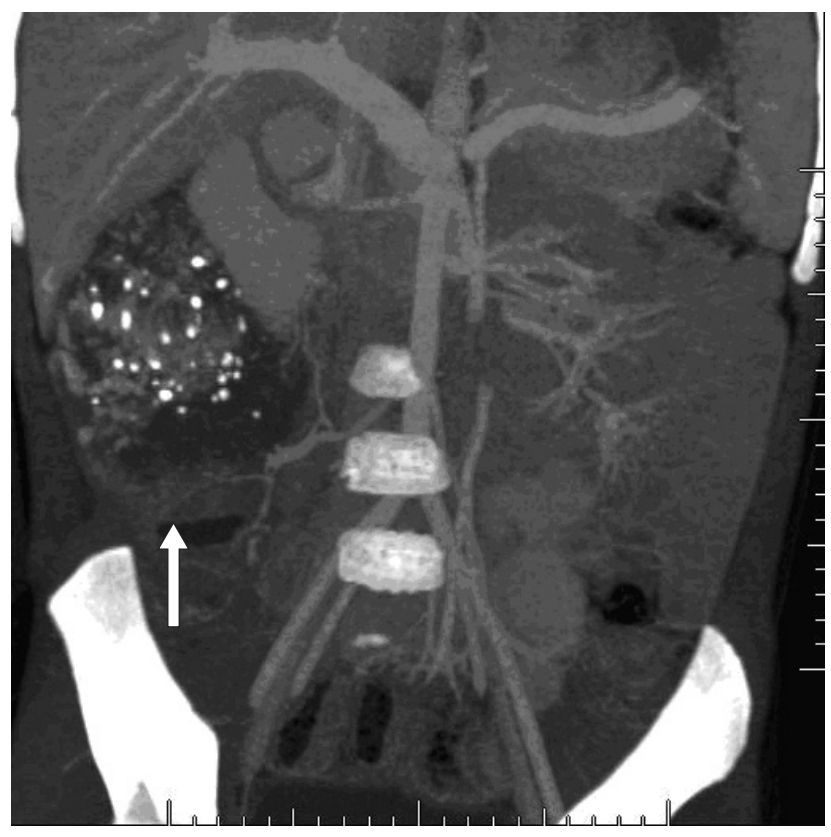

Fig. 3. Hemangioma of proximal ileal loop in 60- yearold man with obscure gastrointestinal bleeding (surgery approved).Coronal MIP reconstruction of arterial-venous phase CT, a lot of punctate calcifications in large mural and extramural enhancing lesion (arrow). excellent soft tissue resolution, its easy comparability for the initial evaluation and the follow-up during the whole life. An MRI unit needs multichannel and phased-array coil, fast gradients, fast and ultrafast gradient echo and steady state sequences. For proper examination patients should fast for at least 5-8 hours and combined with 24 hours the low residue diet before the exam to decrease bowel motion and peristalsis with the resulting blurring artifact. Intraluminal contrast agents can be administered perorally (MR enterography - MREg) or through nasojejunal tube (MR enteroclysis - MREc). Intraluminal contrast agents are essential for lumen opacification and distension, high contrast resolution between the lumen and the bowel wall. Contrast agents have to have minimal mucosal absorption, absence of artifacts, no significant adverse effects and low cost ${ }^{34}$. Numerous contrast agents have been proposed, but there is no consensus on the ideal one $\mathrm{s}^{34}$. There are 3 main groups of intraluminal contrast agents based on provided signal intensity depending on the used pulse sequence. Positive contrast agents (high signal on T2 and T1-weighted images) are gadolinium chelates, manganese chloride, ferrous ammonium citrates, food products ${ }^{34}$. Negative contrast agents (low signal on T2 and T1-weighted images) are solutions with Super Paramagnetic of Iron Oxides (SPIO) including nanoparticles of maghemite in bentonite matrix ${ }^{35}$ (Fig. 4 and 5) Ultrasmall Superparamagnetic of Iron Oxides (USPIO). The third group are biphasic contrast agents (low signal on T1 and high signal on T2-weighted images) like polyethylenglycol (PEG), mannitol/sorbitol, water, methylcellulose (Vidogum), locust bean gum ${ }^{34}$. The dilemma between using oral approach (MREg) which is more acceptable by patients and the intubation-infusion method (MREc) which is less tolerated in the absence of conscious sedation, has not been resolved by the radiologic community ${ }^{36}$. The intubation of the patient and positioning of nasojejunal tube is the most traumatic part of MREc from the patient's point of view. A majority of authors prefer MREg in pediatric population with respect to the more invasive MREc especially in follow-up of inflammatory bowel diseases and their complications ${ }^{37}$.

Use of biphasic contrast agents are preferable ${ }^{38-40}$ in MREc as well as in MREg.

In MREc administration of 1500-2000 $\mathrm{ml}$ of PEG solution through the tube manually or with automatic pump is performed ${ }^{34}$. The infusion rates are the same as in CTEc and should be monitored by ultrafast sequences (MR fluoroscopy). MREg with peroral contrast can be used as a diagnostic tool for evaluation of the small bowel in patients with Crohn's disease and has the potential to replace conventional enteroclysis as follow-up ${ }^{41}$. In the case of MREg with biphasic contrast agent, the patient drinks four 250-450 $\mathrm{ml}$ aliquots of PEG in 50 minutes for a total amount 1000-1800 ml. PEG solution is prepared dissolving granular powder containing $34.8 \mathrm{~g}$ PEG $4000+1.42 \mathrm{~g}$ anhydrous sodium sulphate $+0.42 \mathrm{~g}$ sodium bicarbonate $+0.36 \mathrm{~g}$ sodium chloride and $0.18 \mathrm{~g}$ of potassium chloride in $500 \mathrm{ml}$ of tap water ${ }^{42}$. Another form of biphasic peroral solution is $2.5 \%$ mannitol in total amount $1000 \mathrm{ml}$. In one published study ${ }^{40}$ the authors 


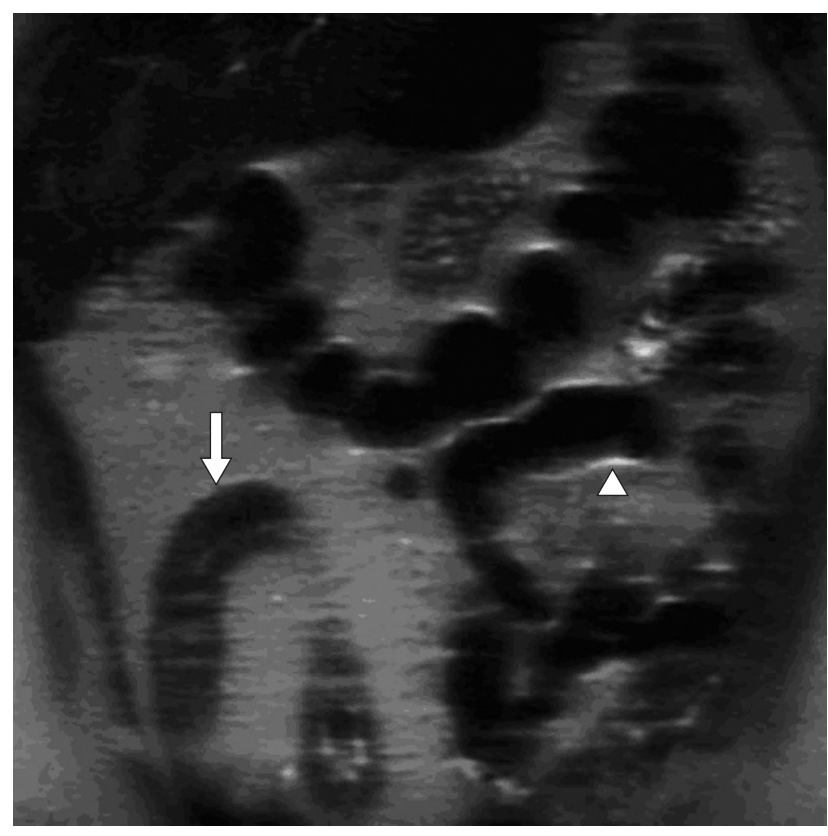

Fig. 4. Active ileal Crohn's disease in 55-year-old-woman. Coronal T2 SSFSE MR enterography with negative luminal contrast agent. Segment of affected loop is without paraluminal fluid/abscess, mural edema is more visible (arrow), homogeneous dark lumen of not affected loops (arrowhead).

compared the bowel distension, acceptance, side effects using the peroral agent - tap water, $0.7 \%$ bulk fiber laxative, $2.5 \%$ mannitol, locust bean gum alone, a combination of $2.5 \%$ mannitol with $0.2 \%$ solution of locust bean gum for optimization of oral MR agent imaging the small bowel. The poorest rate was for water alone, the best rates were for mannitol and locust bean gum in or without combination, inferior was bulk fiber laxative ${ }^{40}$. Differences between locust bean gum and mannitol were less definitive with no statistical significance between combination and the application of mannitol alone ${ }^{40}$. MREg with negative contrast agents uses solution with USPIO or SPIO nanoparticles, including nanoparticles of maghemite in bentonite matrix (Fig.4 and 5) (ref. ${ }^{38}$ ). Negative contrast agents decrease the extent of noise and motion artifacts related to the bowel peristalsis ${ }^{44,45}$ and improve the detection of intramural edema and extramural complications (necrosis, fat edema, mesenterial edema, abscess formation or fistulas). The commercially available negative contrast agents,magnetic iron oxide nanoparticles (Lumirem, Guerbet; Abdoscan, Nycomed) (ref. ${ }^{46,47}$ ) are often used for the peroral bowel MRI diagnostics. They are usually coated with an insoluble material (siloxane, polystyren,etc.) and suspended in viscosity - increasing agent (starch or cellulose) to prevent particles from aggregation and to ensure the homogenous contrast distribution throughout the bowel.The synthesis of these composites is relatively difficult, resulting in their high price and, thus, limiting their use in daily diagnostic practise. Our co-authors describe a new negative contrast agent composed of superparamagnetic nearly monodispersed maghemite nanoparticles incorporated in a layered aluminosilicate mineral (bentonite

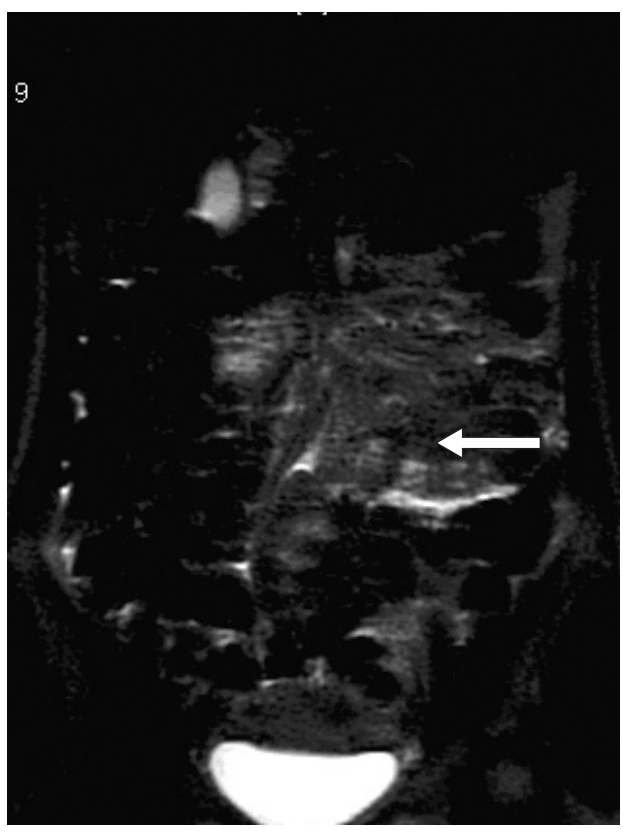

Fig. 5. Celiac sprue (biopsy improved) in 33-year-oldwoman. Coronal T2 SSFSE MR enterography with negative luminal contrast agent, visible mesenterial edema and mural edema in jejunal loops (arrow), extraluminal free fluid, evaluation of mesenterial vessels is impossible.

matrix). Bentonite (montmorillonite clay) as an insoluble matrix is incorporated to the as- prepared maghemite nanoparticles from ferrous acetate on the surfaces of the mineral layers. Bentonite is natural, low-cost, readily available and biocompatible aluminosilicate mineral endowed with unique swelling, sorpsion, ion - exchange and intercalation properties. The clay is known to be essentially nontoxic and nonadsorable, established by the Food and Drug Administration as Generally Recognized As Safe (GRAS) ${ }^{35}$. In clinical test (under surveillance of ethical committee) $1000 \mathrm{ml}$ of contrast suspension is admninistered orally (400 mg maghemite nanoparticles, $4 \mathrm{~g}$ bentonite matrix, $500 \mathrm{ml}$ fruity juice (HAMI apple/

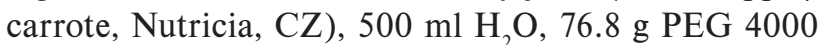
(Makrogol), 2.82 g NaS, 0.84 g NaHCO, 0.72 g NaCl, $0.36 \mathrm{~g} \mathrm{KCl})\left(\right.$ ref. $\left.^{35}\right)$.

Imaging protocol for 1,5 T MR unit (Siemens, GE): patient is in prone position and before examination it is useful to administer intravenously spasmolytics (butylscopolamin, glucagon) for reducing the bowel peristalsis and prolonging small-bowel distension. Scout localizer in 3 planes, transverse and coronal planes in T2 nad T1 sequences as SSFSE (single-shot fast spin-echo) or SSTSE ( single-shot turbo spin-echo) or HASTE (half-Fourier acquired single-shot turbo spin echo), transverse and coronal plane 3D FIESTA (Fast Imaging Employing Steady State Aquistition) or 3D trueFISP (fast imaging with steady-state precession) and then coronal plane fat saturation T1 3D FAME (Fast Aquisition with Multiphase Efgre 3D) or 3D FLASH (fast low-angle shot) or 3D LAVA (Liver Acquisition with Volume Acceleration) / 3D VIBE (Volumetric Interpolated Breathhold Examination) with 


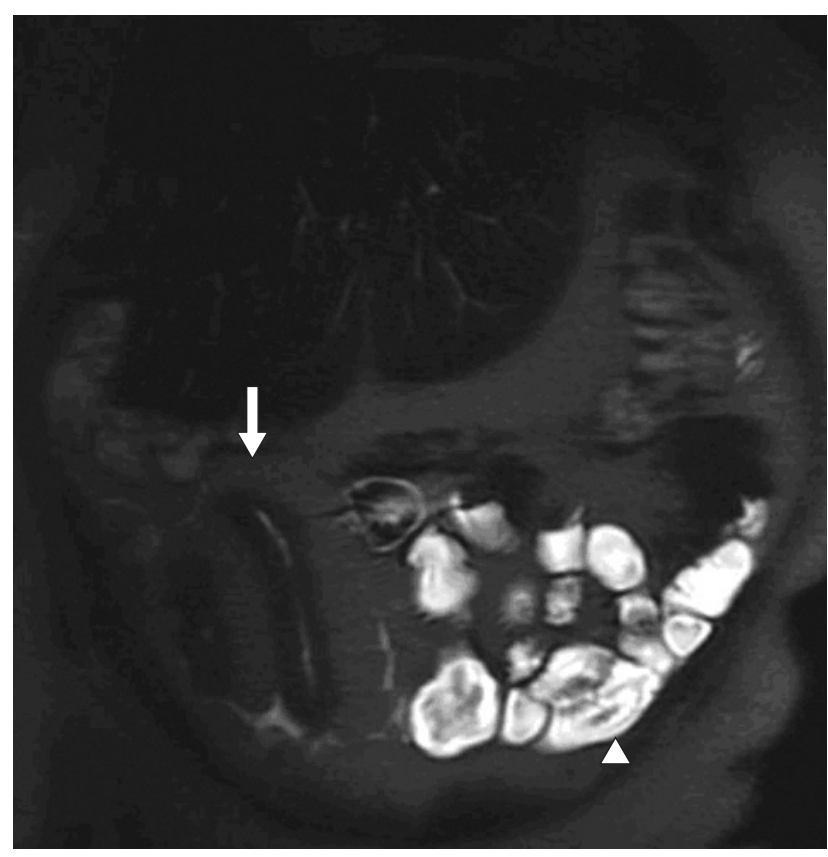

Fig. 6. Active ileal Crohn's disease in 55-year-old woman. Coronal T2 SSFSE MR enterography with biphasic luminal contrast agent. Segmental affected loop of terminal ileum with mural thickening and luminal narrowing (arrow). Small bowel loops without disease (arrowhead).

and without contrast media intravenously. SSTSE is fast sequence appropriated for MR fluoroscopy and provides T2 weighted images. 3D true FISP and 3D FIESTA sequences can provide motion-free high resolution T2 -like images of mesenteries and entire small bowel within few seconds $^{34}$. 3D T1 sequences as FLASH, VIBE, LAVA, FAME, have high spatial resolution, fat saturation prepulses facilitate the demostration of the bowel wall with high signal intensity, after intravenous gadolinium administration increases the conspicuity of the normal bowel wall and may permit lesion characterization by enhancement pattern $^{34}$. HASTE and SSFSE are heavily T2*-weighted images with high contrast resolution, do not suffer from susceptibility or chemical shift artifacts and provide bowel wall with low signal intensity, but they are not appropriate for evaluation of mesenteries (fat edema, vessels, lymph nodes $)^{34}$. The most important clinical application of MREc and MREg is evaluation of Crohn's disease (Figs. 4 and 6), other form of inflammatory diseases, malabsorption syndromes, small-bowel tumors and intestinal obstruction $^{34,48}$. MREc and MREg can demonstrate all abnormalities from the wide spectrum of findings in Crohn's disease, including early non-specific changes such as mucosal nodularity or aphtous-type ulcers, longitudinal or fissure ulcers, cobblestoning, intramural tracts, wall thickening, luminal narrowing and prestenotic dilatation, fibrofatty proliferation, mesenteric hypervascularity, the "comb sign", associated mesenteric lymphadenopathy and/or complications such as fistula formation, phlegmon, abscess and can provide imaging evidence of disease activity.Wall thickening, significant enhancement of mu-

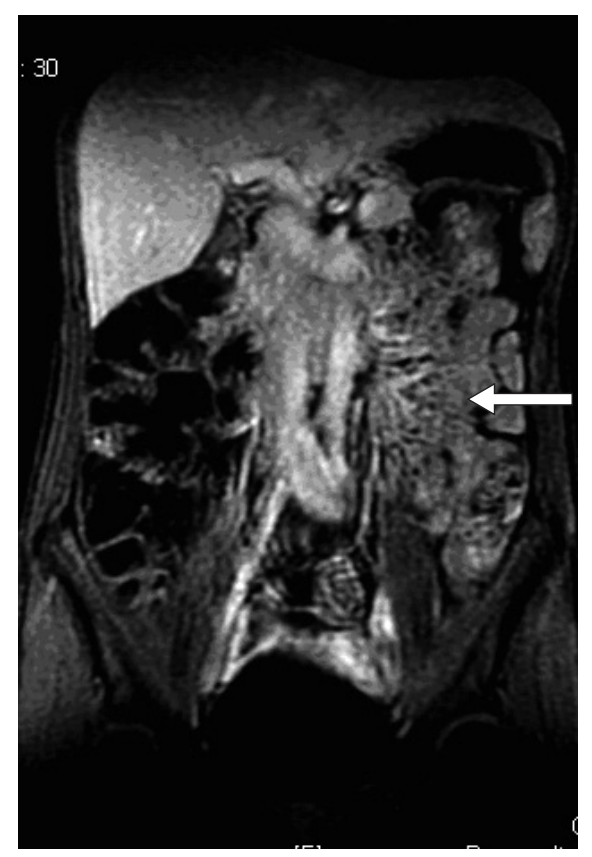

Fig. 7. Celiac sprue (biopsy improved) in 33-year-old woman.Coronal arterial-venous phase gadolinium-enhanced 3D T1- weighted MR enterography with biphasic luminal contrast agent. Prominence of mesenterial vessel, absent of luminal filling in affected jejunal loops (arrow). Ileal loops are without disease.

cosa, relatively hypointense submucosal edema are best seen on postgadolinium images. The severity of disease process can be ranked using the measurements of wall thickening, the length of involved segment can be well appreciated on trueFISP as well as 3DT1 FLASH with fat saturation ${ }^{34}$. Intramural thickening and extraluminal infiltration are appropriately detected on MREc or MREg with no statistically significant difference ${ }^{34}$. Comparing CE and MREc, CE shows more mucosal and mural abnormalities than MREc, but this difference was not statitically significant,but CE was statistically superior to $\mathrm{MREg}^{34}$. Superficial erosions, deep ulcers in the wall, and pseudopolyps were statistically better delineated with MREc than $\mathrm{MREg}^{42}$. No statistically significant differences were found in assessing the diagnostic efficacy between MR examinations for the depiction of mural stenosis and fistulas ${ }^{42}$. The number of detected abscesses, fibrofatty proliferation, lymphadenopathy, and skip enteral/colonic lesions was significantly higher with MREc and with MREg compared to $\mathrm{CE}^{42}$.

Another indication for MREc and MREg are the malabsorption syndrome, Whipple disease and celiac sprue (Fig. 5 and 7), but definitive diagnosis is based on biopsy $^{48}$. Traumatic or congenital diaphragmatic hernia is well depicted especially on MR using sagital and coronal plane.The problem is the timing of the examination in nonstabile traumatic patient ${ }^{49}$.

The role of MREc and MREg in detection of small bowel neoplasms has not been fully established at present, based on the limited experience with these rare tumors ${ }^{34}$. MREc and MREg are very effective in diagnosing small 
bowel obstruction and in revealing the cause of the obstruction - postoperative adhesions, postradiation enteropathy, peritoneal carcinomatosis, Meckel diverticulum, polyps and polyposis syndromes, benign/malignant stenosis.

\section{Endoscopic techniques in small bowel disorders}

Ezofagogastroduodenoscopy (EGD). EGD is the initial test for revealing duodenal disorders such as ulcers, inflammation, reflux, intraluminal tumors, potentional sources of obscure gastrointestinal bleeding (OGIB). Insertion of fiberoptic endoscope through the mouth is comfortable for small bowel biopsies (suspicion of celiac sprue), for miniinvasive procedures (foreign body removal, stricture dilataction, pancreatic pseudocyst drainage, sclerotherapy, argon plasma coagulation). Jejunum and distal part of small bowel are invisible because of the endoscope length $135 \mathrm{~cm}$.

Push enteroscopy.Push enteroscopy is an endoscopic procedure whereby a longer endoscope is inserted into the jejunum through the mouth to evaluate a larger segment of the small bowel. The indications are: looking for the source of OGIB, tumors, inflammation, celiac sprue. This endoscope has biopsy and therapeutic capabality.

Sonde enteroscopy. Sonde enteroscopy involves the use of a long, flexible, fiberoptic instrument propelled through the small bowel by peristalsis, this procedure may allow for viewing the remainder of the small bowel. The sonde instrument relies on a balloon placed at the instrument's tip. Peristalsis then advances the long flexible endoscope to the distal small bowel and the endoscopic examination is performed during withdrawal. In contrast to push enteroscopy this instrument has no biopsy or therapeutic capability. Thus, this procedure is not commonly used because of prolonged procedure time (more than 2 hours) and the inability to perform biopsies or deliver therapy.

Double-balloon enteroscopy. This is a new endoscopic technique for evaluating the small bowel mucosa. This technique allows for more extensive examination of the small bowel and also therapeutic capabilities. The endoscope is $200 \mathrm{~cm}$ in length and has a balloon at the distal end. It also uses a $145 \mathrm{~cm}$ flexible overtube with a balloon and a pump controller to inflate or deflate the balloon. The technique for advancement uses a push and pull method with inflation and deflation of the balloons and telescoping of the intestine onto the overtube. Double-balloon enteroscopy can be used to perform the full range of endoscopic therapies including biopsy, tattooing, stricture dilatation, foreign body removal and argon plasma coagulation. This procedure is also long.

Intraoperative enteroscopy. Intraoperative enteroscopy during laparotomy may be used in patients with suspected OGIB. Endoscopic evaluation has been performed orally, rectally, or through enterostomies at the time of laparotomy.
Ileocolonoscopy. The colonoscope is $200 \mathrm{~cm}$ in the length and it is inserted rectally. Retrograde approach through Bauhin valve to distal ileum allows evaluation of the most distal part of the small bowel. It has biopsy capability.

Wireless capsule enteroscopy (WCE). Although conventional endoscopic examinations i.e. push enteroscopy, ileocolonoscopy, are less sensitive for revealing obscure small bowel lesions, these procedures will remain first line techniques due to their general availability and their provision of simultaneous treatment ${ }^{50}$. Sonde enteroscopy, as a method for examining the entire small bowel, is today replaced by WCE or double-ballon enteroscopy, whereas virtual endoscopies based on new cross-sectional techniques are still advancing. Segments of small intestine that could not be reached by push enteroscopy and retrograde ileocolonoscopy, are now visualized by WCE using biodegradable capsule swallowed by the patient. Capsule follows through and obtains photographic images of small intestine and transmits them via radiofrequency waves to recorded device. The images are downloaded and viewed on computer workstation. WCE detects more abnormalities than small-bowel follow-through (SBFT) and capsule examination is currently undergoing clinical trials. WCE has been shown to be superior to both conventional enteroclysis (CE) and SBFT examination to find ulcerative lesions in patients with gastrointestinal bleeding ${ }^{51}$. However, these techniques only provide indirect information on the intestinal wall and adjacent extraintestinal structures, unlike CT or MRI, which are able to assess mural and extramural findings ${ }^{51}$. WCE system is undergoing rapid technical improvements that may relegate push enteroscopy and intraoperative enteroscopy to those cases in which biopsies or therapy are required. At the same time enteroscopes for total enteroscopy are also improved and may become more widely available, allowing biopsies and therapy in all segments of the small intestine, without the need for operative intervention ${ }^{52}$. OGIB is the foremost indication for WCE. In a pooled analysis, the capsule study found an abnormality in $73 \%$ of patients, while the other methods of investigation revealed only $41.5 \%$ lesions causing the obscure bleeding ${ }^{53}$. For other indications WCE identified lesions not identified by other methods in $52.8 \%$ of patients ${ }^{53}$. There is no radiologic technique to diagnose angioectasia, on WCE it is the common finding explains obscure bleeding ${ }^{53}$. Comparative studies reported in the gastroenterology endoscopic literature have shown that WCE is the test with the highest yield in assessing small-bowel source of bleeding and is superior to SBFT, conventional CT, CT enterography (CTEg). CT enteroclysis (CTEc) or CTEg have a second-line role in the investigation of the probable source of bleeding from the small bowe $1^{51-53}$. Limitations are due to capsule retention, WCE is contraindicated in patients with suspected bowel obstruction or stenosing Crohn's disease, former small bowel surgery, swallowing and motility disorders, intestinal pseudoobstruction, esophageal, small and large bowel diverticula, pacemaker and other electromedical devices. WCE in patients without risk factors that preclude 
the performance of WCE, radiology may be left a limited role and the future will show how the patency capsule will affect the use of radiological investigations ${ }^{53}$.

\section{CONCLUSION}

Small-bowel follow-through (SBFT) has currently only a secondary role in small bowel imaging. Ultrasound and MR enterography are still methods of choice for imaging small bowel diseases in pediatric populations. US and contrast-enhanced ultrasound are also accepted, as a methods of choice, especially in inflammatory cases. Abdominal CT can reveal exactly: congenital lesions, hernias, polytraumatic abdominal conditions, obstructive small bowel diseases, as well as abdominal CT angiography. Conventional enteroclysis is replaced with new cross-sectional imaging techniques CT enteroclysis/ CT enterography or MR enteroclysis/MR enterography. Some authors prefer CT or MR enterographies with peroral bowel preparation and they strictly avoid nasojejunal intubation under fluoroscopic control. CT/MR enterographies techniques are preferred by patients in follow-up of the inflammatory diseases, but radiologic community is not unianimous about their role in the imaging process. MR enteroclysis has better soft tissue contrast, showing it to be far more sensitive in detecting mucosal lesions than CT enteroclysis. CT and MR entroclysis are superior to endoscopic methods in the investigation of small-bowel tumors. Wireless capsule enteroscopy, push enteroscopy and intraoperative enteroscopy give perfect imaging of the mucosal surface of the small bowel in obscure gastrointestinal bleeding and inflammatory diseases. Sonde enteroscopy and double-balloon enteroscopy are not world-wide used techniques and take a lot of time during the examination. Comparing endoscopic and radiologic approach, radiologic techniques are less invasive for patients, they take less time to investigate with the possibility of imaging the entire small bowel. Some of them do not have any radiation exposure (US, MR).Endoscopic methods are not appropriate for congenital lesions, tumoral lesions and traumas, but are preferable as first line for ulcers and bleeding. Endoscopic methods are more expensive, more invasive and need longer examination time, without radiation exposure, but in the majority, there is the possibility of performing biopsy. The current trend is an increase in the radiologic approach.

\section{REFERENCES}

1. Fork FT, Aabakken L. Capsule enteroscopy and radiology of the small intestine. Eur Radiol 2007; 17:3103-3111.

2. Gourtsoyannis N, Papanikolaou N, Grammatikakis J, Maris T, Prassopoulos P. MR enteroclysis protocol optimization: comparison between 3D FLASH with fat saturation after intravenous gadolinium injection and true FISP sequences. Eur Radiol 2001; 11:908-913.

3. Masselli G, Vecchioli A, Gualdi GF. Crohn disease of the small bowel: MR enteroclysis versus conventional enteroclysis. Abdom Imaging 2006; 31:400-409.
4. Makó EK, Mester AR, Tárján Z, Karlinger K,Tóth G. Enteroclysis and spiral CT examination in diagnosis and evaluation of small bowel Crohn's disease. Eur J Radiol 2000; 35(3):168-175.

5. Willis JS, Lobis IF, Denstman FJ. Crohn disease:state of the art. Radiology 1997; 202:597-610.

6. Korman U, Kurugoglu S, Ogut G. Conventional enteroclysis with complementary MR enteroclysis:a combination of small bowel imaging. Abdom Imaging 2005; 30:564-575.

7. Wilson SR. Gastrointestinal Tract. In: Rumack CM, Wilson SR, Charbonaeu WJ,editors. Diagnostic Ultrasound. St.Louis: Mosby; 1998. p. 279-326.

8. Válek VA, Hep A, Všetíček J. Vyšetření tenkého stř̌eva - enteroklýza, CT a ultrazvuk - diferenciální diagnostika patologických stavú. Čes Radiol 2000; 54:59-70.

9. Klibanov AL. Ligand-carrying gas-filled microbubbles: ultrasound contrast agents for targeted molecular imaging. Bioconjug Chem 2005; 16:9-17. Available from: http://en.wikipedia.org/wiki/ Contrast-enhanced_ultrasound

10. Válek V, Kysela P, Vavríková M. Crohn's disease at the small bowel imaging by the ultrasound-enteroclysis. Eur J Radiol 2007; 62(2):153-9.

11. Bender GN, Timmons JH, Wiliard WC, Carter J. Computed tomographic enteroclysis: one methodology. Invest Radiol 1996; 31:43-49.

12. Kohli MD, Maglinte DD. CT enteroclysis in small bowel Crohn's disease. Eur J Radiol 2009; 69:398-403.

13. Romano S, De Lutio E, Rollandi GA, Romano L, Grassi R, Maglinte DD. Multidetector computed tomography enteroclysis (MDCT-E) with neutral enteral and IV contrast enhancement in tumor detection. Eur Radiol 2005; 15:1178-1183.

14. Huprich JE, Fletcher JG. CT enterography: Principles, techniques and utility in Crohn's disease. Eur J Radiol 2009; 69:393-397.

15. Wold PB, Fletcher JG, Johnson CD, Sandborn WJ. Assessment of small bowel Crohn disease: non invasive peroral CT enterography compared with other imaging methods and endoscopy-feasibility study. Radiology 2003; 229:275-28.

16. Horton KM, Eng J, Fishman EK. Normal enhancement of the small bowel: evaluation with spiral CT. J Comput Assist Tomogr 2000; 24:67-71.

17. Sears DM, vots-Avotins A, Culp K, Gavin MW. Frequency and clinical outcome of capsule retention during capsule endoscopy for GI bleeding of obscure origin. Gastrointest Endosc 2004; 60:822-827.

18. Maglinte DD, Bender GN, Heitkamp DE, Lappas JC, Kelvin FM. Multidetector-row helical CT enteroclysis. Radiol Clin North Am 2003;41:249-262.

19. Kalra MK, Maher MM, Toth TL, Schmidt B,Westerman BL, Morgan HT, et al. Techniques and applications of automatic tube current modulation for CT. Radiology 2004; 233:649-657.

20. Voderholzer WA, Beinhoelzl J, Rogalla P, Murrer S, Schachschal $\mathrm{G}$, Lochs $\mathrm{H}$ et al. Small bowel involvement in Crohn's disease: a prospective comparison of wireless capsule endoscopy and computed tomography enteroclysis. Gut 2005; 54:369-373.

21. Solem CA, Loftus EV Jr, Tremaine WJ, Harmsen WS, Zinsmeister AR, Sandborn WJ. Correlation of C-reactive protein with clinical, endoscopic, histologic, and radiographic activity in inflammatory bowel disease. Inflamm Bowel Dis 2005; 11:707-712.

22. Reittner P, Goritschnig T, Petritsch W, Doerfler O, Priedler KW, Hinterleitner T, et al. Multiplanar spiral CT enterography in patients with Crohn's disease using a negative oral contrast material: initial results of a noninvasive imaging approach. Eur Radiol 2002; 12:2253-2257.

23. Booya F, Fletcher JG, Huprich JE, Barlow JM, Johnson CD, Fidler JL, et al. Active Crohn disease: CT Findings and Interobserver Agreement for Enteric Phase CT enterography. Radiology 2006; 241(3):787-95.

24. Bodily KD, Fletcher JG, Solem CA, Johnson D, Fidler JL, Barlow $\mathrm{JM}$, et al. Crohn disease: mural attenuation and thickness at contrast-enhanced CT enterography - correlation with endoscopic and histologic findings of inflammation. Radiology 2006; 238:505-516.

25. Sempere GA, Martinez Sanjuan V, Medina Chulia E, Benages A,Tome Toyosato A, Canelles P, et al. MRI evaluation of inflam- 
matory activity in Crohn's disease. AJR Am J Roentgenol 2005; 184:1829-1835.

26. Winter TC, Ager JD, Nghiem HV, Hill RS, Harrison SD, Freeny PC. Upper gastrointestinal tract and abdomen: water as an orally administered contrast agent for helical CT. Radiology 1996 201:365-370.

27. Horton KM, Fishman EK. Helical CT of the stomach: evaluation with water as an oral contrast agent. AJR Am J Roentgenol 1998; 171:1373-1376.

28. Hara AK, Leighton JA, Heigh RI, Sharma VK, Silva AC, DePetris $\mathrm{G}$, et al. Crohn disease of the small bowel: preliminary comparison among CT enterography, capsule endoscopy, small-bowel followthrough, and ileoscopy. Radiology 2006; 238:128-134.

29. Martin DR, Lauenstein TC, Ascher SM, Semelka RC. Gastrointestinal tract.In: Semelka RC,editor. Abdominal-Pelvic MRI. Hoboken, New Jersey: Willey- Liss; 2006. p.706-742.

30. Boudiaf M, Jaff A, Soyer P, Bouhnik Y, Hamzi L, Rymer R. Smallbowel diseases: prospective evaluation of multi-detector row helical CT enteroclysis in 107 consecutive patients. Radiology 2004; 233:338-344.

31. Maglinte DD, Gage SN, Harmon BH, Kelvin FM, Hage JP, Chua GT, et al. Obstruction of the small intestine: accuracy and role of CT in diagnosis. Radiology 1993; 188:61-64.

32. Walsh D, Bender GN, Timmons JH. Comparison of computed tomography-enteroclysis and traditional computed tomography in the setting of suspected partial small bowel obstruction. Emerg Radiol 1998; 5:29-37.

33. Fukuya T, Hawes DR, Lu CC, Chang PJ, Barloon TJ. CT diagnosis of small-bowel obstruction: efficacy in 60 patients. AJR Am J Roentgenol 1992; 158:765-769.

34. Gourtsoyiannis N, Papanikolaou N, Grammatikakis J, Prassopoulos P. MR enteroclysis: technical considerations and clinical applications. Eur Radiol 2002; 12(11):2651-2658.

35. Kluchova K, Zboril R, Tucek J, Pecova M, Zajoncova L, Safarik I, et al. Superparamagnetic maghemite nanoparticles from solid-state synthesis - Their functionalization towards peroral MRI contrast agent and magnetic carrier for trypsin immobilization. Biomaterials 2009; 30:2855-2863.

36. Maglinte DD, Siegelman ES, Kelvin FM. MR enteroclysis: the future of small-bowel imaging. Radiology 2000; 215(3):639-641.

37. Paolantonio P, Ferrari R, Vecchietti F, Cucchiara S, Laghi A. Current status of MR imaging in the evaluation of IBD in a pediatric population of patients. Eur J Radiol 2009; 69: 418-424.

38. Laghi A, Carbone I, Catalano C, Iannaccone R, Paolantonio P, Baeli I, et al. Polyethylenglycol solution as an oral contrast agent for MR imaging of the small bowel. AJR Am J Roentgenol 2001; 177:1333-1334.
39. Laghi A, Paolantonio P, Iafrate F, Borelli O,Dito L, Tomei E, et al. MR of the small bowel with a biphasic oral contrast agent (polyethylene glycol): technical aspects and findings in patients affected by Crohn's disease. Radiol Med (Torino) 2003; 106(1-2):18-27.

40. Lauenstein TC, Schneemann H, Vogt FM, Herborn ChU, Ruhm SG, Debatin JF. Optimization of Oral Contrast Agents for MR Imaging of the Small Bowel. Radiology 2003; 228:279-283.

41. Schreyer AG, Geissler A, Albrich H, Schölmerich J, Feuerbach $\mathrm{S}$, Rogler G, et al. Abdominal MRI after enteroclysis or with oral contrast in patients with suspected or proven Crohn's disease. Clin Gastroenterol Hepatol 2004; 2(6):491-7.

42. Masselli G, Casciani E, Polettini E, Gualdi G. Comparison of MR enteroclysis with MR enterography and conventional enteroclysis in patients with Crohn's disease. EurRadiol 2008; 18:438-447.

43. Wang Y-XJ, Hussain SH, Krestin GP. Superparamagnetic iron oxid contrast agents: physicochemical characteristic and applications in MR imaging. Eur Radiol 2001; 11:2319-31.

44. Narin B, Aja W, Göhde S, Langhorst J, Akhöz H, Gerken G, et al. Combined small and large bowel MR imaging in patients with Crohn's disease: a feasibility study. Eur Radiol 2004; 14:1535-42.

45. Debatin JF, Patak MA. MRI of the small and large bowel. Eur Radiol 1999; 9:1523-34.

46. Haldeman - Heusler RC, Wight E, Marincek B.Oral superparamagnetic contrast agent (ferumoxsil): tolerance and efficacy in MR imaging of gynecologic disease. J Magn Reson Imaging 1995; 5:385-91.

47. Johnson WK, Stoupis C, Torres GM, Rosenberg EB, Ross RR. Superparamagnetic iron oxide (SPIO) as an oral contrast agent in gastrointestinal (GI) magnetic resonance imaging (MRI): Comparison with state -of -the-art- computed tomography (CT). Magn Reson Imaging 1996; 14:43-9.

48. Laghi A: MRI with or without enteroclysis. In: Postgraduate Educational programme ECR 2009. Book af Abstracts. Eur Radiol Suppl 1/March 2009; 19:28.

49. Ferda J, Kreuzberg B, Mírka H, Novák M, Bosman R, Kučera R. Polytrauma $\mathrm{z}$ pohledu diagnostické a intervenční radiologie.Ces Radiol Suppl 1 2008; 62:64-69.

50. Saurin JC, Delvaux M, Vahedi K, Gaudin JL,Villarejo J, Florent C, et al. Clinical impact of capsule endoscopy: 1-year follow-up study. Endoscopy 2005; 37:318-323.

51. Hara AK, Leighton JA, Sharma VK, Fleisher DE. Small bowel: preliminary comparison of capsule endoscopy with barium study and CT. Radiology 2004; 230:260-265.

52. Lee SD, Cohen RD. Endoscopy of the small bowel in inflammatory bowel disease. Gastrointest Endosc Clin N Am 2002; 12:485-493.

53. Maglinte DD.Capsule imaging and the role of radiology in the investigation of diseases of the small bowel. Radiology 2005; $236: 763-767$ 